\title{
Chromatin Immunoprecipitation
}

National Cancer Institute

\section{Source}

National Cancer Institute. Chromatin Immunoprecipitation. NCI Thesaurus. Code C106048.

A technique that utilizes immunoprecipitation to find protein-DNA interactions in a biological sample. First, the sample is treated causing the formation of protein-chromatin crosslinks. Then, the sample is sheared to separate individual protein-DNA complexes. Finally, an antibody directed against a protein of interest is applied and the protein-DNA complexes are immunoprecipitated. 